\title{
Qualidade de vida de um grupo de idosos vinculados a um plano de saúde na cidade de São Paulo
}

\author{
Quality of life in a group of elderly people attached to a health plan \\ in the city of São Paulo
}

\section{Calidad de vida de un grupo de ancianos vinculados a un plan de salud en la cuidad de São Paulo}

\author{
Henrique Souza Barros de Oliveira $\left(\mathbb{1}\right.$ ', Fernanda Fenner $\mathbb{C}^{2}$, Maria Elisa Gonzalez Manso $(\mathbb{1} \square$ \\ Centro Universitário São Camilo (CUSC). São Paulo, SP, Brasil. \\ 2 Faculdade das Américas (FAM). São Paulo, SP, Brasil.
}

\section{ARTICLE INFO}

\section{Article history}

Received: 02/01/2019

Accepted: 03/05/2019

Published: 29/08/2019

$\triangle$ Correspondent Author

Maria Elisa Gonzalez Manso

Rua Celso de Azevedo Marques, 740/1001

03122-010, São Paulo, SP, Brasil

mansomeg@hotmail.com

\section{(C) 2019 All rights reserved}

\section{Editors}

Alfredo Cataldo Neto

Newton Luiz Terra

Assistant Editors

Paula Engroff

Vanessa Sgnaolin

\begin{abstract}
RESUMO
OBJETIVO: Esta pesquisa teve como objetivo aferir a qualidade de vida de um grupo de idosos vinculados a um plano de saúde na cidade de São Paulo e verificar quais variáveis afetam este construto.

MÉTODOS: Trata-se de uma pesquisa exploratória, transversal, realizada em 2017 com um grupo de idosos, acima de 60 anos e sem déficits cognitivos, vinculados a uma operadora de planos de saúde, todos moradores da cidade de São Paulo, SP. Foram aplicados os questionários auto preenchíveis WHOQOL-BREF e WHOQOL-Old e os idosos participantes responderam a um questionário sociodemográfico, de morbidades e de utilização de serviços desenvolvidos pelos pesquisadores.

RESULTADOS: $O$ grupo pesquisado avalia sua qualidade de vida como boa e está satisfeito com sua saúde, mas alguns pontos se destacam. Para este grupo, morar só, ter doenças cardiocirculatórias ou ter mais de uma doença crônica influenciou negativamente em sua qualidade de vida, enquanto que estar em uma união estável e ter acesso a consultas com intervalos abaixo de seis meses interferiram positivamente. Observouse preocupação do grupo com questões relacionadas à independência, capacidade comunicacional, rede social e com a morte. $\bigcirc$ meio ambiente e a intimidade mostraram-se como aspectos de destaque para este grupo.

CONCLUSÕES: Verificou-se neste grupo de idosos que, o melhor desempenho da qualidade de vida foi obtido nos domínios Meio Ambiente e Relações Sociais e nas facetas Intimidade e Atividades Passadas, Presente e Futuras. Ademais, as variáveis sociodemográficas, de morbidades e de utilização de serviços de saúde afetaram significativamente este construto. As características próprias do grupo pesquisado não permitem generalizar os achados aqui apontados, mas espera-se ter contribuído com o olhar sobre um grupo tão diverso como é o grupo dos idosos brasileiros.
\end{abstract}

DESCRITORES: Qualidade de vida; Idoso; Planos de Saúde.

\section{ABSTRACT}

AIM: This study aims to assess the life quality of a group of elderly people linked to a health plan in the city of São Paulo and to verify which variables affect this construct.

METHODS: This is an exploratory cross-sectional study carried out in 2017 with a group of elderly people over 60 years of age and without cognitive deficits, linked to a health plan operator, all residents of the city of São Paulo, SP. The WHOQOL-BREF and WHOQOL-Old self-filled questionnaires were applied and the elderly participants also answered questionnaires relating to sociodemographic, morbidities and the usage of services developed by the researchers. 
RESULTS: The research group evaluates their quality of life as good and is satisfied with their health, but some points stand out. For said group, living by themselves, having cardiocirculatory diseases or having more than one chronic disease influenced negatively their quality of life, whereas being in a stable unionship and having access to consultations with intervals below six months interfered positively. It was observed that the group was concerned about issues related to independence, communication capacity, social life, and death. The environment and intimacy were shown as highlights for this group.

CONCLUSIONS: It was verified in this group of elderly people that the best quality of life performance was obtained in the Environment and Social Relations domains and in the Intimacy and Past, Present and Future aspects. In addition, sociodemographic, morbidity and health service use variables significantly affected this construct. The characteristics of the research group do not allow us to generalize the findings, but it is hoped to have contributed with the look on a group as diverse as the Brazilian elderly.

KEYWORDS: Quality of life; Elderly; Prepaid Health Plans.

\section{RESUMEN}

OBJETIVO: Esta investigación tiene como objetivo mensurar la calidad de vida de un grupo de personas mayores que están vinculadas a un plan de salud de São Paulo y verificar cuáles son los múltiples componentes que afectan esa construcción.

MÉTODOS: Esta es una investigación exploratoria, transversal, realizada en 2017, con un grupo de personas mayores, todos con edades que ultrapasan los 60 años, sin déficit cognitivos, vinculados a un plan de salud y que viven en la Ciudad de São Paulo, SP. Fueran aplicados los cuestionarios auto rellenables WHOQOL-BREF y WHOQOL-Old y los ancianos participantes también respondieron a un cuestionario sociodemográfico, de morbilidad y de utilización de servicios hecho por los autores.

RESULTADOS: El grupo de personas mayores estudiado analizó la calidad de vida como buena y está satisfecho con su salud, pero algunos puntos se destacan. Para este grupo, las variables vivir solo, tener enfermedades cardiocirculatorias o tener más de una enfermedad crónica influenció negativamente en la calidad de vida, mientras que estar en una unión estable y tener acceso a consultas a intervalos menores que seis meses interfirieron positivamente. Se observó preocupación del grupo con cuestiones relacionadas a la independencia, capacidad comunicacional, red social y muerte. El medio ambiente y la intimidad se mostraron como aspectos destacados para este grupo.

CONCLUSIONES: Se verificó en este grupo de ancianos que el mejor desempeño de la calidad de vida fue obtenido en los campos Medio Ambiente y Relaciones Sociales y en las facetas Intimidad y Actividades Pasadas, Presente y Futuras. Además, las variables sociodemográficas, de morbilidad y de utilización de servicios de salud afectaron significativamente este constructo. Las características propias del grupo investigado no permiten generalizar los hallazgos aquí señalados, pero se espera haber contribuido con la mirada sobre un grupo tan diverso como es el grupo de las personas mayores que viven en Brasil.

Palabras Clave: Calidad de vida; Anciano; Planos de salud de Prepago.

\section{INTRODUÇÃO}

Pode-se pensar qualidade de vida em pessoas idosas como uma interação complexa entre os recursos individuais e o meio ambiente, resultante da relação entre longevidade; saúde; autoestima, autoconceito e autodesenvolvimento; moradia; status social; manutenção dos papeis e qualidade dos vínculos familiares e sociais; renda; acesso; segurança e aprendizado, todos componentes importantes. Pode-se agregar ainda o sentir-se produtivo e contribuir ativamente; ter um propósito e um objetivo, todos estes relacionados com um funcionamento positivo e bem-estar na velhice ${ }^{1-2}$. É um conceito polissêmico, portanto sujeito a múltiplas interpretações, e que varia não só de país para país, mas conforme o período histórico, a cultura, a inserção e estrato social e até individualmente, durante o ciclo de vida do indivíduo ${ }^{3}$.

A Organização Mundial de Saúde (OMS) define qualidade de vida como a percepção do indivíduo acerca de sua posição na vida, de acordo com o contexto cultural e o sistema de valores com os quais convive, e em relação a seus objetivos, expectativas, padrões e preocupações. Ressalta que à medida que $\mathrm{o}$ indivíduo envelhece a qualidade de vida é fortemente determinada por sua habilidade de manter sua autonomia e independência ${ }^{4-5}$.

O conhecimento de quais fatores são mais relevantes para a população idosa na percepção da sua qualidade de vida é fundamental para desenhar estratégias e políticas públicas, já que inclui suas necessidades e preferências no planejamento e avaliação dos serviços sociais e de saúde. Além disto, instrumentos de medição de qualidade de vida podem ser úteis para a avaliação individual do idoso na prática clínica, detectando o impacto de doenças crônicas sobre os indivíduos; verificando a diferença de resultados entre enfermos com gravidades clínicas semelhantes e avaliando prognóstico e efeitos dos tratamentos ${ }^{2}$.

Os instrumentos de avaliação da qualidade de vida em idosos mais frequentemente utilizados no Brasil são World Health Organization Quality of Life Assessment Old (WHOQOL-Old) e World Health Organization Quality of Life Assessment BREF (WHOQOL-BREF), pois encontram-se validados e adaptados ao contexto sociocultural da população idosa brasileira, o que diminui vieses e torna as informações mais acuradas e reprodutíveis ${ }^{6}$.

Esta pesquisa teve como objetivo aferir a qualidade de vida de um grupo de idosos vinculados a um plano 
de saúde na cidade de São Paulo e verificar quais variáveis afetam este construto.

\section{MÉTODOS}

Trata-se de uma pesquisa exploratória e transversal, realizada no período de março a junho de 2017, com um grupo de idosos, com idade igual ou superior a 60 anos, sem déficits cognitivos, vinculados a uma operadora de planos de saúde de pequeno porte, da cidade de São Paulo - SP, Brasil, escolhidos aleatoriamente, por sorteio, dentre os usuários idosos deste plano.

Todos os idosos participantes realizaram previamente testes cognitivos, sendo que nenhum deles possui algum tipo de déficit, visto que ser critério de exclusão da presente pesquisa o ter algum déficit. Ressalta-se que, não houve preenchimento dos instrumentos por familiares, cuidadores ou semelhantes, tendo os idosos respondido aos questionários na frente dos pesquisadores.

A coleta dos dados ocorreu por meio de visitas em domicilio, previamente agendadas por telefone. Durante o contato, o objetivo da pesquisa foi explicitado aos idosos, deixando claro que sua participação era voluntaria e que sua não aceitação não interferiria na atenção prestada pelo plano de saúde. A partir deste passo, as visitas foram agendadas e contabilizou-se 47 anuências e cinco recusas em participar.

Foram aplicados, durante a visita, os questionários auto preenchíveis WHOQOL-Bref e WHOQOL-Old, ambos instrumentos validados e adaptados transculturalmente para a população brasileira, como já mencionado ${ }^{6-8}$

O WHOQOL-Old é um instrumento direcionado a indivíduos idosos, que é composto por 24 questões subdivididas em seis facetas: (1) Faceta Funcionamento dos Sentidos (FS) - avalia o funcionamento sensorial e o impacto da perda das habilidades sensoriais na qualidade de vida; (2) Faceta Autonomia (AUT) referente à independência e autonomia na velhice; (3) Faceta Atividades Passadas, Presentes e Futuras (PPF) - descreve a satisfação sobre conquistas na vida e coisas a que se anseia; (4) Faceta Participação Social (PSO) - delineia a participação em atividades do quotidiano, especialmente na comunidade; (5) Faceta Morte e Morrer (MEM) - relaciona-se a preocupações, inquietações e temores sobre a morte e morrer e, por fim, (6) Faceta Intimidade (INT) - avalia a capacidade de se ter relações pessoais e íntimas ${ }^{6-8}$.

O WHOQOL-Bref, forma abreviada do HOQOL100, é composto por 26 questões, das quais duas referem-se à percepção individual da qualidade de vida pelo respondente, sendo as demais distribuídas em quatro domínios: (1) Domínio Físico; (2) Domínio Psicológico; (3) Domínio Relações Sociais; (4) Domínio Meio Ambiente9.

Ambos os instrumentos, WHOQOL-Old e WHOQOL-Bref, seguem uma escala de Likert para as respostas de todos os domínios e quanto maior a pontuação melhor a qualidade de vida ${ }^{6-8}$. $O$ escore final de cada domínio foi calculado, utilizando uma sintaxe elaborada e recomendada pelo grupo WHOQOL para o software SPSS ${ }^{\circledR}$. De forma geral, cada domínio do instrumento foi representado em escores percentuais de zero a 100 , sendo que, quanto mais próximo de 100 , mais positiva foi a percepção da qualidade de vida do idoso ${ }^{6}$.

Além destes instrumentos, os idosos participantes responderam a um questionário sociodemográfico, de morbidades e da utilização de serviços de saúde desenvolvidos pelos pesquisadores. Este questionário, composto por questões fechadas, permitiu que os escores dos domínios dos instrumentos WHOQOL fossem analisados segundo as variáveis: idade; sexo; estado civil; situação ocupacional; se mora sozinho ou não; a presença de doenças crônicas em tratamento até o momento da entrevista; o período da última consulta médica; presença de internações hospitalares; o hábito de fumar; o consumo de álcool pelo Teste de Identificação de Distúrbio de Uso do Álcool (AUDIT) ${ }^{10}$; sedentarismo; e o Índice de Massa Corpórea (IMC), de acordo com padrões de corte para a idade ${ }^{11}$.

O AUDIT é um instrumento formulado com escala do tipo Likert, validado, adaptados transculturalmente para a população brasileira e largamente recomendado pela OMS para identificação de transtornos devido ao consumo de álcool. Este baseia-se em 10 questões objetivas, de autorrelato, cuja respostas possuem pesos que varia entre zero e quatro pontos. $\mathrm{O}$ escore total indica a classificação de cada indivíduo, sendo "consumo moderado" (de zero e sete), "consumo de risco" (de oito e 15), "consumo nocivo de álcool" (de 16 e 19) e "possível risco para dependência de álcool" (de 20 e 40) ${ }^{10}$.

Para análise, os dados contínuos foram expressos na forma de medidas de tendência central e de dispersão, enquanto que os dados categóricos foram expressos por distribuição de frequência absoluta e relativa. Para a comparação entre os domínios e as variáveis anteriormente especificadas foi utilizado o teste t-Student e nas situações em que em um dos grupos havia poucos participantes $(<10$ idosos) utilizou-se o teste não paramétrico de Mann-Whitney. Para todos os testes foi considerado um nível de significância de $5 \%$, desta forma, foram consideradas diferenças entre os grupos quando $p$-valor foi menor do que $0,05$ ( $p$-valor $<0,05)$. 
O projeto foi aprovado pelo Comitê de Ética em Pesquisa da Pontifícia Universidade Católica de São Paulo (PUC-SP), CAAE nº 70839317.0.0000.5482.

\section{RESULTADOS}

Foram entrevistados 47 idosos vinculados a um plano de saúde da cidade de São Paulo, SP, Brasil. A distribuição entre os sexos mostrou-se quase que paritária, com 53,1\% ( $\mathrm{n}=25)$ de mulheres e $46,9 \%$ $(\mathrm{n}=22)$ de homens idosos. A idade média do grupo era de $72 \pm 6$ anos, variando entre 61 e 90 anos de idade. Observou-se ainda que, a maioria dos idosos da amostra estudada apresentaram 70 anos ou mais $(63,8 \% ; n=30)$.

Quanto à distribuição por estado civil, 72,3\% eram casados ou viviam em situação de união estável e $27,6 \%$ eram viúvos ou solteiros. Sobre a situação ocupacional, 21,3\% referiram não ser aposentados. Ressalta-se que 36 idosos do grupo, ou seja, 81,8\%, não moravam sozinhos. Destes, a maioria citou viver com seus respectivos companheiros ou familiares, e apenas oito idosos relataram morar sozinhos, sendo que um único idoso possuía cuidador.

Todos os idosos entrevistados tinham planos de saúde individuais e a maioria destes pagavam as suas próprias mensalidades (77\%), os demais (23\%) tinham as mensalidades pagas pelos familiares. Salienta-se que, a faixa de renda média/mensal destas pessoas era de $\mathrm{R} \$ 6.000,00$, sendo um mínimo de $\mathrm{R} \$ 3.000,00$ e máximo de $\mathrm{R} \$ 14.000,00$.

Quando avaliadas as principais Doenças Crônicas Não Transmissíveis (DCNT) e fatores de risco associados a estas, a hipertensão arterial mostrou-se a mais prevalente $(63,8 \% ; n=30)$, seguido de dislipidemia $(53,2 \% ; n=25)$, sobrepeso $(36,2 \% ; n=17)$, obesidade $(34 \% ; n=16)$ e diabetes mellitus $(31,9 \%$; $\mathrm{n}=15)$. Encontrou-se ainda, em menor frequência, tireoidopatia, neoplasias, coronariopatia, acidente vascular cerebral (AVC) e osteoporose.

Somente 4,3\% dos idosos deste grupo tinham o hábito de fumar e 57,4\% relataram ser sedentários. Quanto a realização do AUDIT, 23,4\% apresentaram provável risco para dependência.

Todos os idosos incluídos neste estudo faziam acompanhamento médico para tratar as suas doenças, sendo que $48,9 \%$ realizaram, pelo menos, uma consulta nos últimos seis meses e 51,1\% realizaram em até um ano. Em relação a internações hospitalares previas, $57,4 \%$ afirmaram já terem sido internados em algum momento, sendo que 19,1\% foram internados uma vez no último ano e apenas um idoso foi internado duas vezes neste mesmo período.

$\mathrm{Na}$ avaliação da qualidade de vida dos idosos estudados, observou-se que os domínios do WHOQOLBref com maiores escores médio foram: Meio Ambiente $(73,9 \pm 13,4)$ e Relações Sociais $(71,2 \pm 5,4)$, enquanto o menor foi: Físico $(58,2 \pm 9,8)$.

Já em relação ao WHOQOL-Old, as facetas com melhores escores médio foram: Intimidade $(75,6 \pm 22,5)$ e Atividades Passadas, Presente e Futuras $(71,5 \pm 14,8)$; enquanto a menor foi Morte e Morrer $(34,5 \pm 24,9)$. Em relação ao escore total de ambos os instrumentos, o WHOQOL-Bref apresentou pontuação superior ao WHOQOL-Old, conforme visualiza-se na Tabela 1.

Tabela 1 - Escores das facetas/domínios dos instrumentos WHOQOL-BREF e WHOQOL-Old, grupos de idosos vinculados a um plano de saúde, São Paulo - SP, 2017.

\begin{tabular}{|c|c|c|c|c|c|}
\hline & Média & Mediana & Desvio-Padrão & Mínimo & Máximo \\
\hline \multicolumn{6}{|l|}{ Escala WHOQOL-Bref } \\
\hline Domínio Físico & 58,2 & 57,1 & 9,8 & 39,3 & 78,6 \\
\hline Domínio Psicológico & 63,7 & 64,6 & 11 & 37,5 & 83,3 \\
\hline Domínio Relações sociais & 71,2 & 75 & 15,4 & 16,7 & 100 \\
\hline Domínio Meio ambiente & 73,9 & 71,9 & 13,4 & 40,6 & 100 \\
\hline Q1* & 76,7 & 75,0 & 20,2 & 25,0 & 100 \\
\hline $\mathrm{Q} 2 * *$ & 68,9 & 75 & 22,1 & 25 & 100 \\
\hline Escore total & 67 & 66,8 & 10,6 & 36,5 & 89,4 \\
\hline \multicolumn{6}{|l|}{ Escala WHOQOL-Old } \\
\hline Faceta Funcionamento dos Sentidos & 40,6 & 37,5 & 16,8 & 12,5 & 81,3 \\
\hline Faceta Autonomia & 67,2 & 65,6 & 17,1 & 18,8 & 100 \\
\hline Faceta Atividades Passadas, Presentes e Futuras & 71,5 & 68,8 & 14,8 & 31,3 & 100 \\
\hline Faceta Participação Social & 64,8 & 65,6 & 20,2 & 18,8 & 100 \\
\hline Faceta Morte e Morrer & 34,5 & 25 & 24,9 & 0 & 75 \\
\hline Faceta Intimidade & 75,6 & 75 & 22,5 & 0 & 100 \\
\hline Escore total & 59,1 & 58,3 & 10,7 & 34,4 & 88,5 \\
\hline
\end{tabular}

* Q1=Questão 1 do WHOQOL-Bref - Como você avaliaria sua qualidade de vida? ** Q2 = Questão 2 do WHOQOL-Bref - Quão satisfeitos você está com a sua saúde? 
A avaliação das duas questões iniciais do WHOQOL-Bref determinou que $80 \%(n=36)$ dos idosos avaliaram a sua qualidade de vida como boa ou muito boa e $73,4 \%(n=33)$ declararam satisfeitos com a sua saúde.

Com relação a análise comparativa entre as facetas do WHOQOL-Old e variáveis sociodemográficas, encontrou-se diferença significativa entre idosos casados/união estável e faceta Intimidade $(\mathrm{p}=0,018)$; aposentados e faceta Atividades Passadas, Presentes e Futuras $(p=0,044)$ e entre aqueles que realizaram, pelo menos, uma consulta médica no período de seis meses anteriores à pesquisa e faceta Atividades Passadas, Presentes e Futuras $(\mathrm{p}=0,046)$.

Observou-se ainda, que algumas variáveis influenciaram significativamente de forma negativa sobre os escores, tais como: morar sozinho no domínio Relações Sociais $(\mathrm{p}=0,032)$ do WHOQOL-Bref; história previa de coronariopatia na faceta Atividades Passadas, Presentes e Futuras $(\mathrm{p}=0,018)$ do WHOQOLOld; AVC sobre o domínio Relações Sociais $(\mathrm{p}=0,028)$ do WHOQOL-Bref e as facetas Participação Social $(\mathrm{p}=0,028)$, Morte e Morrer $(\mathrm{p}=0,040)$ e no score total $(\mathrm{p}=0,008)$ do WHOQOL-Old; ter mais de três doenças no domínio Relações Sociais $(\mathrm{p}=0,036)$, Físico $(\mathrm{p}=0,021)$, score total $(\mathrm{p}=0,022)$ do WHOQOLBref e facetas Participação Social $(\mathrm{p}=0,001)$, Atividades Passadas, Presentes e Futuras $(\mathrm{p}=0,001)$ e score total $(\mathrm{p}=0,023)$ do WHOQOL-Old. Para as demais variáveis estudadas, não foram encontrados resultados significativos.

\section{DISCUSSÃO}

Orupo pesquisado, apesar de ter um discreto predomínio de mulheres entre os entrevistados, mostrou-se diferente de outras pesquisas com grupos semelhantes, já que análises das características demográficas dos idosos vinculados ao setor de saúde suplementar brasileiro demonstram que as maiores coberturas se referem ao sexo feminino, principalmente na região Sudeste do país ${ }^{12}$.

Quando se observa a idade, porém, o grupo corrobora com a literatura, a qual demonstra que a população vinculada ao setor de saúde suplementar brasileiro se mostra mais envelhecida do que a população brasileira como um todo, as maiores coberturas destes convênios concentrando-se nas faixas etárias acima de 70 anos ${ }^{13-14}$. Para a distribuição segundo o estado civil e ocupação, este grupo de idosos apresenta características concordante com as que são descritas por outros pesquisadores, com predomínio de casados ou em união estável e aposentados, respectivamente ${ }^{12,15}$.
As DCNT representam atualmente a maior causa de morbimortalidade mundial, sendo que $80 \%$ das mortes são atribuíveis à estas causas na cidade de São Paulo $^{16}$. São doenças que aumentam sua prevalência conforme aumenta a idade ${ }^{17,18}$. Comparando-se o grupo pesquisado com outros idosos do setor suplementar, nota-se o padrão de morbidade frequentemente encontrado para este segmento etário, com predomínio da hipertensão, diabetes mellitus, dislipidemias e doenças circulatórias ${ }^{12,15}$.

Nota-se que, neste grupo pesquisado, já são citadas complicações cardiocirculatórias relacionadas à hipertensão, tais como AVC e doenças coronarianas. Outros fatores de risco associados às DCNT também foram encontrados, como tabagismo, etilismo, sedentarismo, sobrepeso e a obesidade. Quando se observa o número de doenças e associações com fatores de risco encontradas para este grupo, notase que não difere de grupos semelhantes. $O$ mesmo pode ser aplicado às consultas realizadas e internações encontradas, o qual repete padrão semelhante a outras pesquisas, tanto com usuários do sistema público quanto de planos de saúde no Brasil112,15, 17-20.

Em relação aos dados encontrados para a qualidade de vida do grupo estudado, entretanto, alguns fatos destacam-se do referido pela literatura para outros grupos de idosos. Ressalva-se que não foram encontradas pesquisas brasileiras utilizando os instrumentos WHOQOL em grupos de pessoas idosas vinculadas à planos de saúde.

Analisando-se as duas questões do instrumento WHOQOL-Bref que versam sobre avaliação da qualidade de vida e a satisfação com a saúde, nota-se que a maioria dos idosos do grupo consideraram boa ou muito boa sua qualidade de vida $(76,7 \% ; n=36)$ e estão satisfeitos com a sua saúde $(68,9 \% ; n=36)$. Nem sempre o indivíduo associa diminuição de habilidades, a incapacidade ou o fato de ter uma ou mais DCNT com insatisfação com a vida e perda de qualidade de vida, fato conhecido como "paradoxo da deficiência"17,19,22-24. Mas, somente estas duas questões analisadas não permitem, isoladamente, inferir como os idosos do grupo avaliam sua qualidade de vida, sendo necessário a análise dos diferentes domínios dos instrumentos WHOQOL aplicados.

Observa-se que os domínios da qualidade de vida com maiores escores, segundo o WHOQOL-Bref, foram: Meio Ambiente $(73,9)$ e Relações Sociais $(71,2)$. Os menores escores referiram-se aos domínios Psicológico $(63,7)$ e Físico $(58,2)$. Em estudos realizados com este instrumento de qualidade de vida em portadores de doenças crônicas, o domínio Físico costuma ter menor pontuação, o que se reputa estar relacionado ao próprio 
processo do envelhecimento, a cronicidade da doença, à necessidade de tratamento contínuo e à presença de complicações, como encontrado neste grupo ${ }^{17,19}$.

Estudos nesta mesma temática realizados com a população geriátrica brasileira, demonstram que o domínio com menor pontuação foi o Meio Ambiente 19-20,22, contrário ao aqui encontrado. Trata-se de um domínio associado com acesso, segurança, recursos financeiros, transporte, ambiente físico, lazer e a socialização. No entanto, o grupo pesquisado, reside em uma grande metrópole e está vinculado a um plano de saúde, cuja características próprias de renda e acesso os distinguem, o que pode explicar a elevada pontuação encontrada contrastando com os demais estudos da literatura.

Quando analisado as facetas do instrumento WHOQOL-Old, encontrou-se maior pontuação nas facetas Intimidade $(75,6)$ e Atividades Passadas, Presente e Futuras $(71,5)$, dados estes também divergentes dos apresentados pela literatura ${ }^{20,22}$. Acredita-se que esta pontuação reflita as próprias particularidades do grupo, onde a maioria dos idosos pesquisados é casado ou vive em união estável, fato corroborado pela análise estatística, com associação significativamente entre a faceta e o estado civil.

Ainda em relação ao WHOQOL-Old, as menores pontuações encontraram-se nas facetas Funcionamento dos Sentidos e Morte e Morrer. Cabe salientar que o próprio processo do envelhecimento prejudica as atividades sensoriais, o que se acentua com o passar dos anos, gerando impacto sobre a qualidade de vida de idosos longevos, como observado neste grupo ${ }^{18,23}$.

Neste estudo algumas associações comparativas de nível significativo foram encontradas. Os idosos que moram só apresentaram menor pontuação no domínio Relações Sociais da escala WHOQOL-Bref, dado preocupante. O domínio Relações Sociais da escala WHOQOL-Bref avalia, além da atividade sexual, o relacionamento social, o apoio de amigos, parentes e comunidade ${ }^{8}$. $O$ isolamento social no idoso está relacionado a uma série de agravos à saúde e deterioração da capacidade funcional22,23. Apesar de muitos idosos preferirem a opção de morar sozinhos, nota-se, neste grupo pesquisado, a necessidade de maior apoio social.

Com frequência, observa-se que a população geriátrica com doenças crônicas pode apresentar diversas associações destas enfermidades, caracterizando a comorbidade, termo designado para o contexto em que uma doença primária está relacionada a outras condições ${ }^{12}$. Interessante notar que os idosos com três ou mais doenças crônicas mostraram sofrer impacto significativo e negativo em diversos domínios avaliados pelos instrumentos WHOQOL. Ressalta-se, principalmente, a influência da presença de comorbidades sobre as atividades que proporcionam convívio social e satisfação sobre conquistas e anseios na vida, o que demonstra que a presença de comorbidades interfere na participação comunitária destas pessoas.

Além disso, encontrou-se, para este grupo de idosos, que todos os domínios/facetas que se relacionam com a independência obtiveram menores pontuações em ambos instrumentos (Físico do WHOQOL-BREF e Função Sensorial e Participação Social do WHOQOLOld).

Outra influência negativa e significativa encontrada foi a associação entre história de doença coronariana e AVC sobre as facetas Atividades Passadas, Presentes e Futuras; Participação social, Morte e Morrer do WHOQOL-Old, respectivamente. Ambos são eventos potencialmente fatais e encontram-se relacionados às internações hospitalares de longa duração, inclusive com admissões e transferências para Unidades de Terapia Intensiva (UTI). Pode-se depreender que estes idosos se preocupam sobremaneira com a possibilidade de um novo evento causar sua morte e demonstram como estas enfermidades tem impacto em sua independênciaa ${ }^{23,25}$. Assim, idosos do grupo com comorbidades e doença circulatória obstrutiva (AVC e coronariopatia) apresentaram pior desempenho em algumas facetas, evidenciando assim, a apreensão destes com o processo de finitude da vida, o seu futuro e a necessidade de medidas de suporte efetiva para melhoria da qualidade de vida.

Interessante ressaltar, em divergência com a literatura ${ }^{17}$, que este estudo não encontrou associação significativa entre as faixas etárias do grupo de pessoas vinculadas ao plano de saúde analisado com os escores dos instrumentos WHOQOL.

Em relação as associações que geraram impacto positivo sobre o desempenho dos idosos, chamamos atenção para os idosos casados, aposentados e que realizam consultas médicas até há seis meses apresentaram maior pontuação nas facetas Intimidades e Atividades Passadas, Presentes e Futuras da escala WHOQOL-Old. Em especial, a frequência das consultas médica foi um achado também descrito em outras pesquisas, nas quais o investimento na saúde física se reflete em melhora na qualidade de vida dos idosos $^{20}$.

\section{CONCLUSÃO}

Conclui-se que, para o grupo de idosos participantes deste estudo, o melhor desempenho da qualidade de vida foi obtido nos domínios Meio Ambiente e Relações Sociais 
e nas facetas Intimidade e Atividades Passadas, Presente e Futuras. Em contrapartida, um pior desempenho foi observado no domínio Físico e na faceta Morte e Morrer. Além disso, este grupo mostrou que fatores como morar só, ser portador de doença circulatória obstrutiva e ter três ou mais doenças crônicas influenciaram de maneira negativa em aspectos da qualidade de vida, enquanto que, ser casado ou ter união estável, ser aposentado e realizar consultas médicas com frequência impactam positivamente na qualidade de vida.

Avaliar a qualidade de vida de idosos auxilia na compreensão da realidade destes, a fim de que estratégias adequadas possam ser desenvolvidas para atendê-los em suas especificidades. Este artigo avaliou a qualidade de vida de um grupo de idosos residentes na cidade de São Paulo e vinculados a um plano de saúde, o que por si só é uma limitação. As características próprias das pessoas pesquisadas não permitem generalizar os achados aqui apontados, mas acredita-se ter contribuído com informações sobre um grupo de idosos ainda pouco estudado no país.

\section{REFERÊNCIAS}

1. Dawalibi NW, Anacleto GMC, Witter C, Goulart RMM, Aquino RC. Envelhecimento e qualidade de vida: análise da produção científica da SciELO. Estud Psicol. 2013;30(3):393-403. https://doi.org/10.1590/s0103 $166 \times 2013000300009$

2. Reis SP, Abrahão GS, Côrtes RM, Carvalho EEV, Abdalla DR, Abdalla GK, Ferreira MBG, Abrahão DPS. Estudo da qualidade de vida de idosos não institucionalizados. JCBS. 2015;1(2):56-60.

3. Paschoal SMP. Qualidade de vida na velhice. In: Freitas EV, Py L,oprganizadores. Tratado de geriatria e gerontologia. 4. ed. Rio de Janeiro: Guanabara Koogan; 2016. p. 147-53.

4. Fleck MPA. A avaliação da qualidade de vida: guia para profissionais de saúde. Porto Alegre: Artmed; 2008. https://doi.org/10.1590/s1413-81232009000600040

5. Centro Internacional de Longevidade Brasil. Envelhecimento ativo: um marco político em resposta à revolução da longevidade. Rio de Janeiro: ILC-BR; 2015.

6. Santos PM. Principais instrumentos de avaliação da qualidade de vida de idosos no Brasil: vantagens e desvantagens na utilização. Corpoconsciência. 2015;19(2):25-36. https://doi.org/10.3895/rbqv.v9n3.5845

7. World Health Organization. WHOQOL: measuring quality of life [Internet]. Geneva: WHO; 1997 [capturado em 30 dez. 2018]. Disponível em: https://www.who.int/ mental health/media/68.pdf.

8. Fleck MP, Chachamovich E, Trentini CM.

Development and validation of the Portuguese version of the WHOQOL-Old module. Rev Saúde Pública. 2006;40 (5):785-91. https://doi.org/10.1590/s003489102006000600007
9. Fleck MPA, Louzada S, Xavier M, Chachamovich E, Vieira G, Santos L, Pinzon V. Aplicação da versão em português do instrumento abreviado de avaliação da qualidade de vida "WHOQOL-Bref". Rev Saúde Pública. 2000;34(2):178-83. https://doi.org/10.1590/s003489102000000200012

10. Portugal. Serviço de Intervenção nos Comportamentos Aditivos e nas Dependências (SICAD). Instrumentos de rastreio e intervenção da rede [Internet]. Lisboa: SICAD; 2015. [capturado em 30 dez. 2018]. Disponível em: http://www.sicad.pt/PT/Intervencao/RedeReferenciacao/ SitePages/Instrumentos.aspx. https://doi.org/10.14195/ 978-989-26-0866-2

11. Pascali C, Peliello LC. Avaliação Nutricional. In: Manso MEG, Biffi ECA, editores. Geriatria: manual da LEPE. São Paulo: Martinari, 2015. p. 91-104.

12. Manso MEG, Osti AV, Borrozino NF, Maresti LTP. Avaliação multidimensional do idoso: resultados em um grupo de indivíduos vinculados a uma operadora de planos de saúde. Rev Kairós. 2018; 21(1): 191-211.

13. Veras RP, Caldas CP, Araújo DV, Kuschnir R, Mendes W. Características demográficas dos idosos vinculados ao sistema de saúde suplementar de saúde no Brasil. Rev Saúde Pública. 2008;42(3):497-502. https://doi. org/10.1590/s0034-89102008005000024

14. Leite F. Envelhecimento populacional e a composição etária de beneficiários de planos de saúde. São Paulo: IESS. 2011.

15. Manso MEG, Câmara R, Souza AS, Maciel TD, Farina DBL. Programa de gerenciamento de doenças crônicas em um plano de saúde, São Paulo, Brasil. Cienc Cuid Saúde. 2016;15(2):321-7. https://doi.org/10.4025/cienccuidsaude. v15i2.28683

16. Malta DC, Stopa SR, Szwarcwald CL, Gomes NL, Silva Júnior JB, Reis AAC. A vigilância e o monitoramento das principais doenças crônicas não transmissíveis no Brasil - Pesquisa Nacional de Saúde, 2013. Rev Bras Epidemiol. 2015;18(suppl 2):3-16. https://doi.org/10.1590/1980 5497201500060002

17. Tavares DMS, Côrtes RM, Dias FA. Qualidade de vida e comorbidades entre os idosos diabéticos. Rev Enferm UERJ. 2010;18(1):97-103.

18. Diniz MA, Tavares DMS. Fatores de risco para doenças cardiovasculares em idosos de um município do interior de Minas Gerais. Texto Contexto Enferm. 2013; 22(4):885-92. https://doi.org/10.1590/s0104-07072013 000400003

19. Pereira DS, Nogueira JAD, Silva CAB. Qualidade de vida e situação de saúde de idosos: um estudo de base populacional no sertão central do Ceará. Rev Bras Geriatr Gerontol. 2015;18(4):893-908. https://doi. org/10.1590/1809-9823.2015.14081

20. Tavares DMS, Matias TGC, Ferreira PCS, Pegorari MS, Nascimento JS, Paiva MM. Qualidade de vida e autoestima de idosos na comunidade. Ciênc Saúde Coletiva. 2016;21(11):3557-64. https://doi.org/10.1590/ $1413-812320152111.03032016$ 
21. Cruz MF, Ramires VV, Wendt A, Mielke GI, MartinezMesa J, Wehrmeister FC. Simultaneidade de fatores de risco para doenças crônicas não transmissíveis entre idosos da zona urbana de Pelotas, Rio Grande do Sul, Brasil. Cad Saúde Pública. 2017;33(2):e00021916. https://doi. org/10.1590/0102-311×00021916

22. Peixoto N, Lima LCV, Bittar CML. Percepções sobre qualidade de vida entre idosos que participam de uma Universidade Aberta para Maturidade. Acta Scientiarum. 2017;39(2):209-16. https://doi.org/10.4025/ actascihumansoc.v39i2.33089

23. Serbim AK, Figueiredo AEPL. Qualidade de vida de idosos em um grupo de convivência. Scientia Med. 2011;21(4):166-72.

24. Chachamovich E, Trentini CM, Fleck MP. Qualidade de vida em idosos: conceituação e investigação. In: Neri AL, organizador. Qualidade de vida na velhice: enfoque. Campinas: Alínea; 2007. p. 61-81. https://doi.org/10.3895/ s2175-08582010000200004

\section{Scattolin FAA, Diogo MJD, Colombo RCR.}

Correlação entre instrumentos de qualidade de vida relacionada à saúde e independência funcional em idosos com insuficiência cardíaca. Cad Saúde Pública. 2007;23(11):2705-15. https://doi.org/10.1590/s0102$311 \times 2007001100018$

\section{CONFLITOS DE INTERESSE:}

Os autores deste artigo declaram não haver conflitos de interesse.

\section{AUTHORS:}

HeNRIQUe SOUZA BARROS DE OliveIRA

Médico. Centro Universitário São Camilo - CUSC, São Paulo, SP, Brasil.

E-mail: heeenry.barros@icloud.com

Orcid: http://orcid.org/0000-0002-9067-8581

FERNANDA FENNER

Graduanda do curso de Medicina, Faculdade das Américas (FAM), São Paulo, SP,

Brasil.

E-mail: fernandafenner88@gmail.com

Orcid: https://orcid.org/0000-0002-9012-8731

MARIA ELISA GONZALEZ MANSO

Médica. Pós-doutorado em Gerontologia Social, Doutora em Ciências Sociais e Mestre em Gerontologia Social pela Pontifícia Universidade Católica de São Paulo (PUC-SP). Master em Psicogerontologia pela Universidade Maimônides, Buenos Aires, AR. Docente do curso de Medicina do Centro Universitário São Camilo (CUSC-SP). Docente orientadora das Ligas Acadêmicas LEPE (Liga de Estudos do Processo do Envelhecimento do Centro Universitário São Camilo SP) e LAISI (Liga Acadêmica Interdisciplinar de Saúde do Idoso da Faculdade das Américas SP).

E-mail: mansomeg@hotmail.com

Orcid: https://orcid.org/0000-0001-5446-233X 